\title{
IV.
}

\section{FORTGESETZTE NACHRICHTEN}

von den Verfuchen des Uhrmachers Degen in Wien

mit feiner Flugmaschine, จ०॥

Johand Christoph Stelzhammer, Director des k. k. phylikalifchen Kabiuetts in Wien.

(In einem Schreiben an den Prof. Gilbert in Halle.)

Wien den 8. Decemb. 1808.

Meine Ihnen gegebene Zurage, die Nachrichten uber die Flugmafchine des Uhrmachers Degen, welche im Septemberhefte Ihrer Annalen [XXX. St. 9. S. 1.] ftehn, fortzufetzen, erfülle ich mit defto mehr Vergnügen, da ich in Ilırem Schreiben den patriotifchen Ausdruck finde: dafs Sie Degens Beftreben, als ein nationales Unternehmen, einer Auszeichnung würdig finden.

Die Schrift des Herrn Degen ift feitdem erfchienen; ich lege für Sie ein Exemplar derfelben bei. Hätte Gich der Künftler die Zeit genommen, die Theile der Mafchine einzeln zu entwerfen, fo hätte frch in ihr vieles abkürzen laffen; da er aber an feiner Flugmafchine alles allein arbeitete, fo befchäftigte ihn diefes fo fehr, dafs ihm dazu keine 


\section{$\left[\begin{array}{ll}19^{3} & ]\end{array}\right.$}

Zeit blieb, und ich habe von ihm nur durch wiederlioltes Fragen ganz befriedigende Antworten über die einzelnen befchriebnen Stucke erhalten können *).

Aus dem nemlichen Grunde haben fich dio Vorftellungen, welche der Kunftler in freier Luft, in Vereinigung feiner Mafchine mit einem LuftbalJon geben wollte, fo lange verzögert. Alles, was dazu gehörte, beforgte er allein, und fo konnte er nicht vor dem November zu der erften Vorftellung

*) Befchreibung einer newen Flugmafchine von Jak ob De. gen, burgerlichert Uhrmacher. Mit einer Kupfertafet. Wien 1808. VIII. 39 S. I. In 47 Paragraphen wird die Mafchine in ihren einzelnen Theilen, die Ablicbt des Künfrlers bei feinen Vorrichtungen, und der llechanismus beim Fliegen mit derfelben fo dentlich befchrieben, dafe Hr. Abbé Scelzh ammer allen Anfpruch anf den Dank der Freunde der Phylik, für diefe miihevolle Arbeit hat, auch wenn - wie Herr Prechtel in Brünn, in feinem Auf. fatze über den Flug der Vögel, im vorigen Novemberhefre die. Ser Anralen (XXX. St. 1.1.), darzuthuu fucht, - die Degrn. Sche Flugmalchine noch fehr weit von der Vollkommen. heit entfernt ift, die fich iht durch Nachbildung der Flug. werkzeuge der Vögel hätte geben laflelt. Dér Weg, den Herr Prechtel bei felnen Unterfuchungen über das Flie. gen.mit grüudlicher Einficht eingefchlagen hat, wird ihn uultreitig fehr viel weiter als den Wiener Küufter führen; doch dürfe dem, der eine Flugmalchine, fo wie er fie entwerfen wird, ausführt, immer noch ein Verdienle übrig bleiben; und daher fcheint mir der erfte einigerma fsen glück. liche Verluch, mit Flügeln zu fiegen, der allgemeinen Theilnahne dex deufchen Pubikums, und der Belohnung niche unwiirdig zu feyn, welche Herru $D$ egen in Wien reich!ich 2u Theil werden foll. (Die beiden Vorfte!lungen am 13. und 15. November haben ibm nach öffentlichen Blät. tern Jo000 Fl. eingebracht.)

Anaal. d. Pby Gik. B. 3t. St. 2. J. I\$Og. St.2. N

Gilberc. 


\section{[ 194 ]}

fchreiten. Er kündigte fie in feinen Anfchlag Zetteln auf den 4. November an, -in dem Prater, auf demfelben Platze, wo die Feuerwerke gegeben werden. Es wurde indefs eine vorläufige Probe anbefohlen, und nach ihr die erfte Vorftellung verfchoben, wegen des Windes, wie auf den Ab. fage-Zetteln ftand. So kam erft am 13 . November die erfte Vorftellung $z u$ Stande. Am 15 . November wurde fie wiederholt. Noch follte eine dritte Vurftellung in Gegenwart Sr. Majeftät des Kaylers erfolgen; der Winter trat aber ein, ehe fie gegeben werden konnte.

Sio wünfchten in Ihrem Briefe an mich zweierley: erftens dafs die Maafse des Luftballons und das Gewicht aller Theile genau angegeben wurden; und zweitens dafs wir Beobachtungen mit Mefsinftrumenten aber die Höhe anftellen möchten, bis zu welcher der an dem Ballon fchwebende Künfiler fich erheben würde.

Der erfte diefer Wünfche war fchon in der Befchreibung erfültt. Sie finden in dem Anhange $z u$ derfelben alles, was Sie in diefer Hinficht intereffiren wird. Der Tafft zu dem Ballon (402 Wiener Ellen) war fo forgfältig gewebt und mit Firnifs getränkt, und die Nähte waren damit fo genau überzogen worden, dafs, als der Ballon mit gemeiner Luft aufgeblafen in dem Redoutenfaale hing, er in eilf Tagen nur $\frac{x}{7}$ der eingefchlofsnen Luft durch den Druck der Halle verlor. Die Geftalt war eine vollkommne Kugel, aus $3_{2}$ fphäri- 


\section{[ 19.5$]$}

fchen Sectoren zufammengefetzt. Der Durchmeffer diefer Kugel betrug $19^{\prime} 5^{\prime \prime}$, der Umfang eines gröfsten Kreifes derfelben $6{ }^{\prime}{ }^{\prime}$; ihre Oberfäche 1184 Quadratfufs, und ihr körperlicher Inhalt 3833 Kubikfufs. So viel atmofphärifche Luft wiegt $23 y_{2}^{\frac{x}{2}}$ Pfund, wenn man den Kubikfufs zu 1 Unze rechnet; und nehmen wir an, dafs das Wafferftoffgas, womit der Ballon gefült wurde, fechsmahl leichter war, fo hätte das Gewicht deffelben fehr nahe 40 Pfund betragen. Die ganze Hülle wog nur $19 \frac{x}{3}$ Pfund, und das Sicherheitsventil noch $\frac{x}{2}$ Pfund. Das letztere war am obern Pol der Kugel angebracht, und beftand aus $36 \mathrm{La}$ gen dünen dreimahl mit Leinwand durchfchoffenen Papiers, das zufammengeleimt, dann gefirnifst, und mit dünem Leder uberzogen worden war; der Ring hatte $10^{\prime \prime}$, die Oeffnung deffelben $4 "$, und die nach unten fich öfnende mit einem meffingnen Charnier verfehene Klappe 6" im Durchmeffer; die Schnur ging von diefer Klappe durch eine luftdicht fchliefsende Halfe im untern Pole durçh, und war an der Bruft der Fliegenden bes feftigt; wenn er fie zog, fo öffnete fich die Klappe fo weit, dafs fie unter einem rechten Winkel ftand; fobald das Ziehn nachliefs, fchlofs fie fich vermöge einer Feder, die beim Oeffnen aufgerollt wurde. Das Netz beftand aus lichtblauen feidnen Bändern, jedes aus 140 Fäden gewebt, und beftimmt ein Ge. wicht von 5 Pfund zu tragen; fie gingen von einem $7^{\prime \prime}$ weiten Ring aus $2^{\prime \prime \prime}$ dickem Fifchbein aus, an 


\section{[ $\left.\quad{ }_{19} 9^{6}\right]$}

welchem zuglęich der Ring des Ventils mit 8 Schnüren feft gebunden war, und endigten fich in $3_{2}$ feidne Schnüre, jede $1 \frac{\pi}{2}$ Linie dick und 15 Fuls lang, an einem Ringe aus Fichtenholz, der $2^{\prime} 6^{\prime \prime}$ weit und $2^{\prime \prime}$ hoch war. Von diefem gingen 8 feidne, 3 Linien dicke Schnure herab; vier derfelben verbanden den Luftball mit dem Sattel zwifchen den Beinender Fliegenden; die vier andern waren dazu beftimmt, den Ballon vor und nach dem Verfuch zurück zu halten. Das ganze aus 2200 Ellen Band beftehende Netz wog nur 2 Pfund 10 Loth, und der Reif mit den daran hängenden Schnüren $3 \frac{x}{2}$ Pfund.

Diefes giebt folgende Rechnung: Gewicht der Halle, des Ventils, des Netzes und der Schnüre $2 \overline{0} \frac{1}{\xi}$ Pfund; Gewicht des Kinftlers und feiner Flugm,fchine zufammengenommen 144 Pfund. Wenn man den Ballon vollkommen aufgeblafen bütte, und das Wafferftoffgas in ihm fechsmahl leichter als die atmolphärifche Luft gewefen wäre, fo würde der Ballon an der Erde eine Steigkraft von ${ }_{17} 7 \frac{4}{5}$ Pfund gehabt, folglich diefe feine Steigkraft das Gewicht der Fliegenden und der Flugmalchine um $3, \frac{4}{5}$ Pfund aberwogen haben. Es kam indels nur darauf an, dafs der Ballon den Kilnftler uber die Bäume erhoben erbielt, ohne ihn aus der Nälıe der Zufchauer allzuweit zu entrücken.

So viel ron dem Luftballon. Was den zweiten Ihrer Wunfche betrifft, fo bin ich nacb meinen Kräften bemuht gewefen, demfelben nach zu kom- 


\section{$\left[\begin{array}{lll}197 & ]\end{array}\right.$}

men, und genaue Beobachtungen über die Höhe, bis zu welcher der Künftler fich erheben würde, zu veranlaffen.

Der junge Graf Rezewuski und die Freiherrn von $T a b e r n a t$ und von $L i c h t e n f t e r n$ haben als Kenner und Beförderer von wiffenfchaftlichen Unternehmungen diefer Art, ihre Theodulithen dazu mit aller Bereitivilligkeit angeboten; und mehrere haben fich diefem Gefchäft unterzogen, welche mit allen Kenntniffen dazu ausgerüftet und gehörig geubt find. Herr Oberft Richter vom kaiferl. königl. Generalftab erbot fich zur Leitung diefer Meffungen, und wählte das Invalidenhaus an der Landftrafse zu feinem Standpunkte; Herr Artillerie-Lieutenant Pödot $\mathbf{c} \mathbf{b}$ ning ein Haus an der Donau zu Erberg, und der Civit-Feldmeffer Hr. Brägl, ein junger fehr gefchickter Mann, ein Haus an der Franzbrücke. Von allen diefen Orten aus kann man den obern Theil des Geruftes far die Hauptdekorationen der Feuerwerke fehn, das bis zur Windfabne 117 Wie-ner Fufs hoch ift, und man hatte die Entfernungen jener Oerter von diefem Punkte vorläufig nach dem Plane von Wien und von den Vorftädten, welchen man für den richtigften hält, gemeffen. Der Kunftfeuerwerker Stuver zeigte durch Signale auf diefem Gerüfte den Zeitpunkt an, wenn der Ballon aufftieg, und wenn er nach feiner Schätzung die grölste Höhe erreicht hatte. Die beiden Verfuche des Herrn Degen am 13. und am 15. No. 


\section{$\left[\begin{array}{lll}1 & 1 & 8\end{array}\right]$}

vember gingen indefs fär zwei diefer Standpunkto verloren. Der erfte Verfuch wurde (wegen einer anbefohlnen vorläufigen Probe) allzu kurze Zeit zuvor angekúndigt, und mit dem zweiten trafen Amtsgefohăfte zufammen; fobald indefs eine dritte Vorftellung zu Stande kōmmt, hoffen wir diele Meflungen vollftändig anzuftellen. Beide Mahl kounte nur Herr B ragl beobachten; er hatte dazu das erfte Mahl den Thurm der Karmeliter in der Leopoldftadt, das zweite Mabl das oben erwähnte Haus an der Franzbrücke gewählt. Nach den gemachten Berechnungen erhob fich Degen das erfte Mahl auf $40 \mathrm{Klafter}$, das zweite Mahl, nachdem er Ballaft weggegeben hatte, auf 105 Klafter.

Bei diefen beiden Vorftellungen wurde der Luftballon, die Vorbereitungen abgerechnet, jedes Mahl in 6 Stunden gefüllt, und zwar aus 7 Fäffern. Jedes. Fafs konnte 12 Wiener Eimer in fich faffen. Für eine Füllung wurden genommen 9 Centner fogenannte Nordhäufer Vitriolfaure, 7 Centner Bohrfpäne aus Gufscifen, und auf jedes Fafs $5 \frac{\pi}{2}$ Centner Waffer. Der nicht ganz vollgefüllte Luftballon hob vor beiden Vorftellungen 152 Pfund, wie dieles Gewichte bewiefen, die man an die Spitze des Kegels, welchen die vom Balle herabhangenden feidenen Schnüre bildeten, anbing und fo lange vermehrte, bis fie den Künftler, der mit Jeiner Flugmalchine $144 \mathrm{Pfund}$ wog, mit der Luft in das Gleichgewicht fetzten. Als diefes gefchehn, und allo alle Steigkraft des Ballons aufgehoben 


\section{$\left[\begin{array}{lll}199 & ]\end{array}\right.$}

war, nahm der Künftler noch in einem an feiner Bruft bängenden Beutel aus Tafft Bleyfchrot mit, deffen er fich in der Höhe, ohne jemanden zu befchädigen, entledigen konnte. Deffen ungeachtet vermochte er fich durch die Kraft feiner Flügel zuheben. Diefes, und dafs er fich nach Willkir fenken und wieder erheben konnte, überzeugto jedermann, dals ihn der Ball allein nicht zu heben im Stande war.

Von dem Sicherheitsventil machte der Künftler keinen Gebrauch, wenn er fich nach kleinen Erhebungen wieder fenkte. Es wurde zum erften Mahle geöffnet, als er fich bei feiner zweiten Vorftellung auf der gröfsten Höhe befand. Der Himmel war damals mit dichtem Nebel überzogen; Herr Degen fühlte fich benetzt, und vermutheto einen plötzlichen Regen, weshalb or zu diefem Hülfsmittel fchneller herab zu fteigen fchritt. Der Beobachter, der die Hähe mit dem Theodolithen nahm, hatte die Mitte des Balles in feinem Fernrohre, Er wendete fein Auge was, und las die Zahlen am, Vernier des Inftrumentos; als er wieder in fein Fernrohr fah, fand er den Luftball noch mitten im Geficbtsfelde, und während er am Vernier befchäftiget war, beobachtete ein Neber.ftehender den Ballon durch das Fernrohr. Nach geöffnetem Ventile fank der Ballon in einer etwas gekrümmten Richtung zu der rechten Seite der Gallerie herab, von der man die Feuerwerke anzufehen pflegt, und der Künftler inachte fich von feiner Mafchine los. Die länglichte Geftalt, in 


\section{$[200]$}

welche fich die Kugelform umgeāndert hatte, bewies, wie fehr viel Gas der Ballon durch das Oeffnen des Ventils verlohren hatte.

Folgendes waren die Bewegungen, welche der Kinftler, in der Luft fchwebend, durch Hilfe feiner Flugmafchine vornehmen konnte, und die ich felbft beobachtete, und uber die ich, um aller Täufchung zu entgehen, zugleich Andere befragt habe. Er konnte fich nach Willkür lenken, wenn er, ohne allen Schlag, feine Flugel wagerecht, ausgefpannt bielt. Das Sinken gefchah dann fo langfam, dals keinen Zufeher dabei eine Furcht uberfiel, und dafs er fogar etwas ausruhen konnte, um fich mittelft neuer Flügelfchläge wieder zu erheben. Der Kinftler konnte fich ferner in der Luft umwenden, wenn er eine der Handhaben vorwärts, die andere mehr rückwärts ergriff. Ja, er konnte fich auch ohne Flügelfctlag von dem Luftftrom felbft erheben laffen, wenn er den einen Fliggel aufwärts, den andern ahıvärts, beide mit ibrer untern eingebogenen Fläche dem Luftftrome entgegen hielt. Da feine Mafchine Anfangs nur für den fenkrechten Flug, aus Urfachen, die ich in der Befchreibung angebe, gebantet war, fo konrite er dio mehr horizontale Bewegung in freier Luft nicht in das Werk fetzen; die Richtung im Ganzen war die des Luftftromes. Bei dem allererften Probeverfuche, zu welchem dem Luftballe noch Fullung abging, herrfchten Windfiöfse; lie machten fich in dem zu wenig gefullten Balle eine Ver- 


\section{[ 201$]$}

tiefung, und wirkten dadurch fo heftig auf ibn, dafs fich befürchien liefs, der Ball möchto noch ror leiner weitern Erhebung an die Baume, die den Felverwerksplatz umgeben, angetrieben, und die fo mahfam gebaute Mafchine befchädiget werden. Es wurde daber befoblen, dafs bei den beiden offentlichen Vorftellungen, zur Sicherheit eine nber $100 \mathrm{Klafter}$ lange Schnur, (die noch mit einer Hanffchnur bei dem zweiten Verfuche verlangere wurde), an den Ball gebunden, und von einem Gehulfen gehalten werden mulste.

Der ftarken Bewegung des Kunftlers, die er mit Hand und Fufs zugleich vorzunehmen hatte, mag es zuzufcbreiben feyn, dafs er keinen merklichen Unterfchied der Temperatur bemerkt zu haben angiebt. Das Thermometer ftand an der k. k. Sternivarte am 1\%. auf $+4^{\circ}$ Reaumar; das Barometer auf 28 Zoll 7 Linien; die Windrofe zeigte Nordweft, fchwach. Am 15. ftand das Thermometer auf $+2^{\circ}$, das Baroineter auf $28 \mathrm{Zoll}$ 7 Linien 8 Punkten, der Zeiger an der Windrofo ftand auf Weftnordweft, und beinahe in Ruhe.

Der Kunftler kam beide Mahl ohne alle Befchädigung zur Erde, und obfchon fich ein fchwaches Gerinche verbreiten wollte, dafs der Verfuch feiner Gefundheit einigen Nachtheil zugezogea habe, fo liahen fich doch alle, die nachher mit ithm zufammentrafea, ganz vom Gegentheile überzeuge 


\section{$\left[\begin{array}{lll}202 & 0\end{array}\right]$}

gefunden. Die verfchiedenen Sagen von der anbefohlenen Probe, nach welcher die erfte Vorftellung verfchoben wordea war, und die Anfchlagzettel, auf welchen, der entftandenen Winde wegen, die Vorftellung ain 4. November abgefagt wurde, hatten bei Manchem ein ungünftiges Urtheil erregt. Defto gultiger war die allgemeine Zufriedenheit, die man laut bezeugte, und mit der man von beiden Vorftellungen fprach.

Der Kanftler erwartete die Ankunft Sr. Majeftät des Kailers, um noch in liefem Jahre eine dritte Vorftellung zu geben. Se. Majeftät, zurickgehalten in Ollmutz durch die Krankheit Ihrer Majeftät der Kaiferin, kamen endlich glücklich an; allein fchon am nämlichen Tage war das Land mit Schnee uberzogen, auf welchen anhaltende Nebel und fturmifche Witterung folgte.

Zu diefem dritten Verfuche la le ich jetzt einen Aëroftat aus Gold[chlägerhäutchen verfertigen; einen Merkur in Lebensgröfse, der unter den Augen des berahmten Bildhauers und Profeffors an der Akademie der bildenden Künfte, Martin Fifcher, über einer Statue geformt wird; diele kunfteiche Figur foll vor der Vorftellung auffiegen. Vor der zweiten Vorftellung ivurden 2 kleine taffetne Bälle losgelaffen. Der eine, kugelfürmige, war von dem nämlichen Stoffe als der grofse Luftballon gemacht, und batte 3 Fufs, 9 Zoll im Durch. 


\section{[ 203$]$}

meffer. Er erhob fich bis in die Wolken, und kam 4 Strafsen - Meilen von Wien zur Erde zuruck. Dem zweiten hatte ich die länglichte Form eines Fifches geben laffen, um den Aufhängungspunkt aufser dem Mittelpunkte anbringen, und beobach. ten zu können, welchen Einflufs diefes auf feine Bewegung haben werde. So lange er noch an der Schnur zurückgehalten wurde, machie er wellenförmige Bewegungen; als ex losgelaffen wurde, ftieg er ebenfalls fetir hoch, und ging, wie es der Beobachter durch das Fernrohr des Theodoliths bemerkte, in Schraubenlinien durch die höhern Luftchichten fort. 